\title{
Correlation between Magnetic Resonance Imaging (MRI) Findings with Histological Grading of Patients with Supratentorial Diffusely Infiltrating Astrocytomas
}

\author{
Hasan $\mathrm{MM}^{1}$, Kadir $\mathrm{ML}^{2}$, Uddin $\mathrm{KH}^{3}$, Rana $\mathrm{MS}^{4}$, Khan $\mathrm{UKS}^{5}$, Hossain $\mathrm{MM}^{6}$, Hafiz $\mathrm{AM}^{7}$, Islam $\mathrm{MS}^{8}$, \\ Mukherjee $\mathrm{SK}^{9}$, Hossain $\mathrm{MA}^{10}$
}

Conflict of interest: There is no conflict of interest relevant to this paper to disclose.

Funding Agency: was not funded by any institute or any group.

Contribution of Authors: Principal Investigator and Manuscript preparationData collection-

Scalp block with anaesthesia-

Editorial formatting-

Copyright: @2020bang.BJNS published by BSNS. This article is published under the creative commons CC-BY-NC license. This license permits use distribution (https://creativecommons. orgf/licences/by-nc/4-0/)reproduction in any medium, provided the original work is properly cited and is not used for commercial purposes.

Received: 01.03.19

Accepted: 28.06.19

\begin{abstract}
Data regarding the impact of MRI for diagnosis and staging of brain tumors in our population is sparse. Thus, the purpose of this study is to determine the efficacy of MRI in preoperative diagnosis of primary intra-axial brain tumors and to determine its diagnostic accuracy in grading of gliomas in our population, correlated with histopathological (hematoxylin \& eosin stain) findings taking as gold standard. There has been extensive works in developed countries regarding the diagnostic effectiveness of MRI. But only a few works have been done in our country. To be very particular, little work has been done regarding the performance tests of MRI regarding histological correlation with gliomas in Neurosurgery Department, Bangabandhu Sheikh Mujib Medical University, Dhaka, Bangladesh. So, I have tried to correlate MRI findings with the histopathological (hematoxylin \& eosin stain) findings of supratentorial diffusely infiltrating astrocytomas. To evaluate the relationship of Magnetic Resonance Imaging (MRI) findings with histological grading of patients with gliomas, to assess MRI findings of gliomas and to assess histological grading of gliomas. The study was carried out in the Department of Neurosurgery, Bangabandhu Sheikh Mujib Medical University, Dhaka, Bangladesh, from March 2015 to August 2016. This study was descriptive type of observational study (Cross sectional study). Study Population were include all the patients with supratentorial gliomas admitted in the department of neurosurgery, Bangabandhu Sheikh Mujib Medical University, Dhaka, Bangladesh, during the above mentioned time. All patients were admitted through out patient \& emergency department of Neurosurgery, Bangabandhu Sheikh Mujib Medical University, Dhaka, Bangladesh, were included in the study after primary screening with inclusion and exclusion criteria. History of the patients were taken with demographic data. General examinations were carried out after admission. Neurological examinations were carried out thoroughly. Grading of gliomaswere done from MRI findings. Histopathologies were done of resected tumour tissues. After ethical clearance for the study from the Department of Neurosurgery and IRB, Bangabandhu Sheikh Mujib Medical University, Dhaka, Bangladesh, written informed consents were taken from the patients and/or the legal guardians/responsible family members after completely explaining the procedure and the purpose of the study in easy local language. Patients data were collected in data collection sheets. Every patient was enjoy every right to participate or refuse participation and had the right to withdraw from the study at any time without compromising their medical care. The privacy of the patients were strictly maintained and the patients informations were notdisclosed to any source. The study data were onlyused for the purpose of this scientific study. Data were processed and
\end{abstract}

1. Dr. Md. Motasimul Hasan, Assistant Professor, Neurosurgery, Dhaka Medical College, Dhaka, Bangladesh.

2. Dr. Md. Lylatul Kadir, Assistant Professor, Neurosurgery, Rajshahi Medical College, Rajshahi, Bangladesh.

3. Dr. Kazi Hafiz Uddin, Assistant Professor, Neurosurgery, National Institute of Neurosciences, Dhaka, Bangladesh.

4. Dr Md Sumon Rana, Assistant Professor, Neurosurgery, Dhaka Medical College, Dhaka, Bangladesh.

5. Dr Uzzal Kumer Sadhu Khan, Assistant Professor, Neurosurgery, Dhaka Medical College, Dhaka, Bangladesh.

6. Dr Muhammad Mahabub Hossain, Assistant Professor, Neurosurgery, Sir Salimullah Medical College, Dhaka, Bangladesh.

7. Dr Ahsan Mohammad Hafiz, Assistant Professor, Neurosurgery, Uttara Adhunik Medical College, Dhaka, Bangladesh.

8. Dr Md Shafiqul Islam, Associate Professor, Neurosurgery, Dhaka Medical College, Dhaka, Bangladesh.

9. Dr Sudipta Kumer Mukherjee, Associate Professor, Paediatric Neurosurgery, Dhaka Medical College, Dhaka, Bangladesh.

10. Professor M Afzal Hossain, Professor \& Chairman (Ex), Neurosurgery, Bangabandhu Sheikh Mujib Medical University, Dhaka, Bangladesh.

Address of Correspondence: 
analyzed using computer software SPSS (Statistical Package for Social Sciences) version 22.Descriptive data presented on categorical scale were expressed as frequencies and corresponding percentages, while the data presented on continuous scale were measured as mean and standard deviation (SD). Correlation between categorical variables were evaluated using Spearman's rank correlation test. For analytical tests, level of significance was set at 0.05 and p-value $<0.05$ was considered significant. The summarized informations were then presented in form of tables and figures. For the validity test, sensitivity, specificity, accuracy, positive predictive value and negative predictive value of MRI in detecting the grade of intracranial gliomas were calculated after confirmation of the diagnosis by histopathology.

Results: The age range of patients were 18 to 70 years. The peak age incidence were $>50$ years age group. $75.8 \%$ patients were male and $24.2 \%$ patients were female. The male to female ratio was 3.12:1. Regarding midline crossing in MRI of brain , 24 (72.7\%) patients showed no crossing of midline, 2 (6.1\%) patients showed equivocal and $7(21.2 \%)$ patients showed crossed midline. In 4 (12.1\%) patients there were mild edema, 12 (36.4\%) patients showed moderate edema and 17 (51.5\%) patients showed marked edema. 10 (30.3\%) patients had well circumscribed lesion, 15 (45.5\%) patients had poorly circumscribed lesion and 8 (24.2\%) patients had highly infiltrating lesion. 7 (21.2\%) patients showed mild mass effect, 18 (54.5\%) patients had moderate mass effect and 8 (24.2\%) patients had severe mass effect. Necrosis/cystic change of tumor were present in 19 (57.6\%) cases. 6 (18.2\%) patients showed mild heterogeneity, 22 (66.7\%) patients showed moderate heterogeneity and 5 (15.2\%) patients showed severe heterogeneity. Definitive haemorrhage showed $5(15.2 \%)$ patients. WHO grading of gliomas by MRI. 17 (51.5\%) patients of the gliomas were classified as WHO Grade IV, followed by 9 (27.3\%) patients were Grade II and 7 (21.2\%) patients were Grade III. WHO grading of gliomas by histopathology. 16 (48.5\%) patients of the gliomas were classified as WHO Grade IV, followed by 9 (27.3\%) patients were Grade II and 8 (24.2\%) patients were Grade III. Out of 33 patients, 09 patients were diagnosed as grade II (Low grade gliomas) by MRI, among them 08 cases were proved as grade II (Low grade glioma) and 01 case was grade III (Anaplastic Astrocytoma) by histopathology. 07 patients were diagnosed as grade III (Anaplastic Astrocytoma) by MRI, among them 05 cases were proved as grade III (Anaplastic Astrocytoma) and 01 case was grade II (Low grade glioma) and 01 case was grade IV (Glioblastoma Multiforme) by histopathology. 17 patients were diagnosed as grade IV (Glioblastoma Multiforme) by MRI, among them 15 cases were proved as grade IV (Glioblastoma Multiforme) and 02 cases were (Anaplastic Astrocytoma) by histopathology. Among 33 patients, 09 patients were diagnosed as low grade gliomas by MRI, among them true positive were in 08 cases and 01 cases were diagnosed as low grade gliomas by MRI but was diagnosed as high grade gliomas by histopathology. This 01 case was false positive. Out of 33 cases of gliomas 24 were diagnosed as high grade gliomas by MRI, among them 23 cases were true negative and 01 case was diagnosed as high grade gliomas by MRI but was proved as low grade gliomas by histopathology. This 01 case was false negative. MRI is accurate in preoperative diagnosis and assessing the characteristics of intracranial gliomas. It is very accurate in assessing the grade of gliomas. Tumor necrosis, irregular margins, mass effect of tumor and peritumoral edema are most important indicators of tumor grade. The present study revealed that there is significant correlation between the MR imaging features and histopathological grading of intracranial gliomas.

Bang. J Neurosurgery 2020; 10(1): 67-74

\section{Introduction:}

The term glioma was first used by Virchow as he described the neuroglia as the interstitial matrix of the brain in which the individual cells are suspended. Glioma is the general name for the tumors that arise from the glial cells of the brain ${ }^{1}$.

Diffusely infiltrating astrocytomas are the most common intracranial neoplasms accounting for $>60 \%$ of all primary brain tumors. They occur in 6 instances per 100,000 individuals per year and are slightly more common in males than females. They can arise at any site in the central nervous system and are predominant in the cerebral hemisphere ${ }^{2}$.

Most brain tumors remain asymptomatic during early development, revealing their symptoms and lethal nature only at later stages. Therapy is facilitated many 
times by an earlyfinding, a circumstance making the neuroimaging approaches particularly useful in the detection and handling of these lesions ${ }^{3}$.

In low-grade gliomas, seizure is a common presenting symptom. Subtle changes in neurologic function including sensation, vision, speechor motor changes may also be present early in lowgrade gliomas. Alternatively, this may be an incidental finding in certain cases and symptom progression may be insidious. Higher-grade

gliomas typically present with increased neurologic deficits, seizures and symptoms and signs of increased intracranial pressure. The symptoms in these cases can progress at a faster pace. The clinical course of glioblastoma is generally short and less than 3 months in more than half of the cases, whereby the patient presents with the above symptoms that have rapidly progressed ${ }^{2}$.

For a long time, the histogenesis and classification of CNS tumors have been the almost exclusive domain of surgical pathologists whose main focus was the establishment of clinicopathologic correlations which would guide decision making regarding adjuvant radiotherapy or chemotherapy and allow the prediction of clinical outcome. In recent years, tumors of the nervous system have become a focus of basic research ${ }^{4}$.

Prompt diagnosis and treatment of cerebral neoplasms are critical to decrease both morbidity and mortality. $\mathrm{CT}$ and MRI are the mainstays of imaging in current practice $^{5}$.

Among the various imaging modalities used in clinical practice, MRI, by virtue of its availability, contrast versatility, pathophysiologic specificity and potential for repeat studies without adverse effects on the health of subjects is often the method of choice. MRI provides excellent soft tissue contrast and is particularly powerful for imaging of the brain ${ }^{6}$.

In the last decades, Magnetic Resonance Imaging (MRI) approaches have evolved into the most powerful and versatile imaging tool for brain tumor diagnosis, prognosis, therapy evaluation, monitoring of disease progression and planning of neurosurgical strategies. MRI methods enable the non invasive assessment of glioma morphology and functionality providing apoint of likeness into histopathological grading of the tumor and helping in this way a more successful patient management ${ }^{3}$.
Astrocytomas are histologically heterogeneous group, having varying degrees of cellular and nuclear pleomorphism, mitotic activity, vascular proliferation and necrosis ${ }^{7}$.

Astrocytomas are classified into three grades; lowgrade astrocytoma, anaplastic astrocytoma and glioblastoma multiforme. Accurate grading of astrocytoma is critical for planning therapeutic strategies, assessing prognosis and monitoring of response to therapy ${ }^{8}$.

A difficulty in the management of astrocytomas is related to potential sampling errors and improper grading with needle biopsy. This is due to focal areas of more malignant features widespread among regions with a less aggressive histopathological appearance. Diagnosis and staging mainly rely on imaging

features. Tumor necrosis, irregular margins and peritumoral edema are most important indicators of gliomas type as well as astrocytomas grade. It can help early diagnosis, staging, grade assessing, preoperative planning for surgical resection and post operative management plan. MRI have significant role in characterizing and staging of these tumors ${ }^{5}$.

There has been extensive works in developed countries regarding the diagnostic effectiveness of MRI but only a few works have been done in our country in this regard. To be very particular, little work has been done regarding the performance tests of MRI regarding histological correlation with gliomas in Neurosurgery Department, Bangabandhu Sheikh Mujib Medical University, Dhaka, Bangladesh. So, I have tried to correlate MRI findings with the histopathological (hematoxylin \& eosin stain) findings of diffusely infiltrating astrocytomas. Histopathological findings was considered as gold standard. Finally the relationship of Magnetic Resonance Imaging (MRI) findings with histological grading of gliomas were evaluated.

\section{Method}

It was a cross sectional observational study. This study was carried out at Department of Neurosurgery, Bangabandhu Sheikh Mujib Medical University, Dhaka, Bangladesh. The study was carried out during the period of March 2015 to August 2016. Purposive sampling according to availability of the patients and strictly considering the inclusions and exclusions criteria. Only patients having histopathologically proven intracranial gliomas who underwent surgery or 
stereotactic biopsy were considered as a study population. Data were collected from Department of Neurosurgery, Bangabandhu Sheikh Mujib Medical University, Dhaka, Bangladesh. Sample size was 33. The inclusion criteria were Patients with intracranial astrocytomas diagnosed by MRI findings, underwent surgery or stereotactic biopsy for intracranial astrocytomas, proven a case of gliomas by histopathological reports and agreed to be included in the study. Exclusion criteria were brain tumors other than astrocytomas by histopathological report, Astrocytomas where MRI were not done, those cases who did not undergo operation or stereotactic needle biopsy hence histopathology report was not available and who refused to be included in the study. Appropriate data were collected by using a preformed data sheets. All other needed data were collected from history sheets and investigation papers. All histopathology reports were collected. Variables studied (a)Demographic variables, such as Age and sex of the patients ; (b) MRI Features/Findings such as Crossing mid line,Peri-lesional edema, Signal heterogeneity,Tumor hemorrhage,Tumor border definition, Necrosis/cystic changes of tumor and mass effect of tumor; (c) MRI Grading such as Low-grade astrocytoma, Anaplastic astrocytoma, Glioblastoma multiforme $^{9}$; (d) Histopathological variables such as Histopathological grading (WHO grading). Data were processed and analysed using computer software SPSS (Statistical Package for Social Sciences) version 22. Descriptive data presented on categorical scale were expressed as frequencies and corresponding percentages, while the data presented on continuous scale were measured as mean and standard deviation (SD). Correlation between categorical variables were evaluated using Spearman's rank correlation test. For analytical tests, level of significance was set at 0.05 and $p$-value $<0.05$ was considered significant. The summarised informations were then presented in form of tables and figures. For the validity test sensitivity, specificity, accuracy, positive predictive value and negative predictive value of MRI in detecting the grade of intracranial astrocytomas were calculated after confirmation of the diagnosis by histopathology.

\section{Result:}

The age range of patients were 18 to 70 years. The mean \pm SD age was $41.7 \pm 14.7$. The peak age incidence were $>50$ years age group. Shown in Table No. I
4.1 Distribution of patients by age

Table-I

Distribution of patients by age $(n=33)$

\begin{tabular}{lcc}
\hline Age (years) & Frequency $(\mathrm{n})$ & Percentage $(\%)$ \\
\hline $\mathrm{d}$ "20 & 2 & 6.1 \\
$21-30$ & 7 & 21.2 \\
$31-40$ & 6 & 18.2 \\
$41-50$ & 8 & 24.2 \\
$>50$ & 10 & 30.3 \\
Total & 33 & 100 \\
Mean \pm SD & $41.7 \pm 14.7$ & \\
Range (min-max) & $18-70$ & \\
\hline
\end{tabular}

In this study, out of 33 patients of gliomas 25 (75.8\%) patients were male and $8(24.2 \%)$ patients were female. The male to female ratio was $3.12: 1$. The sex distribution of the patients is shown in figure 1

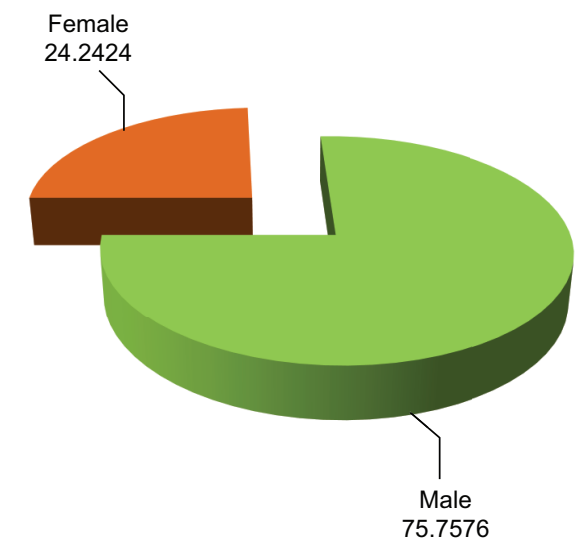

Fig.-1: Pie-chart showing distribution of patients by $\operatorname{sex}(n=33)$

Table II demonstrates the distribution of patients by their MRI features. Regarding crossing midline, 24 $(72.7 \%)$ patients showed no crossing of midline, 2 (6.1\%) patients showed equivocal and $7(21.2 \%)$ patients showed crossed midline. In 4 (12.1\%) patients there were mild edema, $12(36.4 \%)$ patients showed moderate edema and $17(51.5 \%)$ patients showed marked edema. $10(30.3 \%)$ patients had well circumscribed lesion, 15 (45.5\%) patients had poorly circumscribed lesion and $8(24.2 \%)$ patients had highly infiltrating lesion. $7(21.2 \%)$ patients showed mild mass effect, $18(54.5 \%)$ patients had moderate mass effect and $8(24.2 \%)$ patients had severe mass effect. Necrosis/cystic change of tumor were present in 19 $(57.6 \%)$ cases. $6(18.2 \%)$ patients showed mild heterogeneity, $22(66.7 \%)$ patients showed moderate heterogeneity and $5(15.2 \%)$ patients showed severe 
heterogeneity. Definitive haemorrhage showed 5 $(15.2 \%)$ patients.

4.2 Distribution of patients by MRI features of gliomas

Table-II

Distribution of patients by MRI features

of gliomas $(n=33)$

\begin{tabular}{lcc}
\hline MRI features & Frequency & Percentage \\
\hline Crossing midline & & \\
No crossing of midline & 24 & 72.7 \\
Equivocal & 2 & 6.1 \\
Crossed midline & 7 & 21.2 \\
Peri-lesional edema & & \\
Mild edema & 4 & 12.1 \\
Moderate edema & 12 & 36.4 \\
Marked edema & 17 & 51.5 \\
Heterogeneity & & \\
Mild & 6 & 18.2 \\
Moderate & 22 & 66.7 \\
Severe & 5 & 15.2 \\
Tumor hemorrhage & & \\
No hemorrhage & 27 & 81.8 \\
Equivocal & 1 & 3.0 \\
Definitive hemorrhage & 5 & 15.2 \\
Tumor border definition & & \\
Well circumscribed lesion & 10 & 30.3 \\
Poorly circumscribed lesion & 15 & 45.5 \\
Highly infiltrating lesion & 8 & 24.2 \\
Mass effect & & \\
No mass effect & & \\
Mild to moderate mass effect & 18 & 54.5 \\
Marked mass effect & 8 & 24.3 \\
Necrosis/Cystic change of tumor & \\
No & 11 & 33.3 \\
Equivocal & & \\
\hline Present & & \\
\hline
\end{tabular}

WHO grading of gliomas by MRI. 17 (51.5\%) patients of the gliomas were classified as WHO Grade IV, followed by $9(27.3 \%)$ patients were Grade II and 7 (21.2\%) patients were Grade III.
Shown in Table No. III

4.3 Distribution of patients by WHO grading of gliomas by MRI

Table-III

Distribution of patients by WHO grading of gliomas by MRI ( $n=33)$

\begin{tabular}{lcc}
\hline WHO grading & Frequency & Percentage \\
\hline Grade II & 9 & 27.3 \\
Grade III & 7 & 21.2 \\
Grade IV & 17 & 51.5 \\
\hline Total & 33 & 100.0 \\
\hline
\end{tabular}

Patients by WHO grading of gliomas by histopathology. $16(48.5 \%)$ patients of the gliomas were classified as WHO Grade IV, followed by 9 (27.3\%) patients were Grade II and $8(24.2 \%)$ patients were Grade III. Shown in Table No. IV

4.4 Distribution of patients by $\mathrm{WHO}$ grading of gliomas by histopathology

Table-IV

Distribution of patients by WHO grading of gliomas by histopathology $(n=33)$

\begin{tabular}{lcc}
\hline WHO grading & Frequency & Percentage \\
\hline Grade II & 9 & 27.3 \\
Grade III & 8 & 24.2 \\
Grade IV & 16 & 48.5 \\
\hline Total & 33 & 100.0 \\
\hline
\end{tabular}

Table IV shows out of 33 patients, 09 patients were diagnosed as grade II (Low grade gliomas) by MRI, among them 08 cases were proved as grade II (Low grade glioma) and 01 case was grade III (Anaplastic Astrocytoma) by histopathology. 07 patients were diagnosed as grade III (Anaplastic Astrocytoma) by $\mathrm{MRI}$, among them 05 cases were proved as grade III (Anaplastic Astrocytoma) and 01 case was grade II (Low grade glioma) and 01 case was grade IV (Glioblastoma Multiforme) by histopathology. 17 patients were diagnosed as grade IV (Glioblastoma Multiforme) by MRI, among them 15 cases were proved as grade IV (Glioblastoma Multiforme) and 02 cases were (Anaplastic Astrocytoma) by histopathology. 
4.5 Comparison between histopathological diagnosis and MRI diagnosis of patients of gliomas

\section{Table-V}

Comparison between histopathological diagnosis and MRI diagnosis patients of gliomas $(n=33)$

\begin{tabular}{|c|c|c|c|c|}
\hline \multirow[t]{2}{*}{ MRI findings } & \multicolumn{3}{|c|}{ Histopathological diagnosis } & \multirow[t]{2}{*}{ Total } \\
\hline & Grade II & Grade III & Grade IV & \\
\hline Grade II & 8 & 1 & 0 & 9 \\
\hline Grade III & 1 & 5 & 1 & 7 \\
\hline Grade IV & 0 & 2 & 15 & 17 \\
\hline Total & 9 & 8 & 16 & 33 \\
\hline
\end{tabular}

Correlation of MRI diagnosis with Histopathological diagnosis of diffusely infiltrating supratentorial astrocytomas. Shown in figure No. II

4.6 Spearman's rank correlation test for correlation of MRI diagnosis with Histopathological diagnosis of diffusely infiltrating supratentorial astrocytomas $(n=33)$

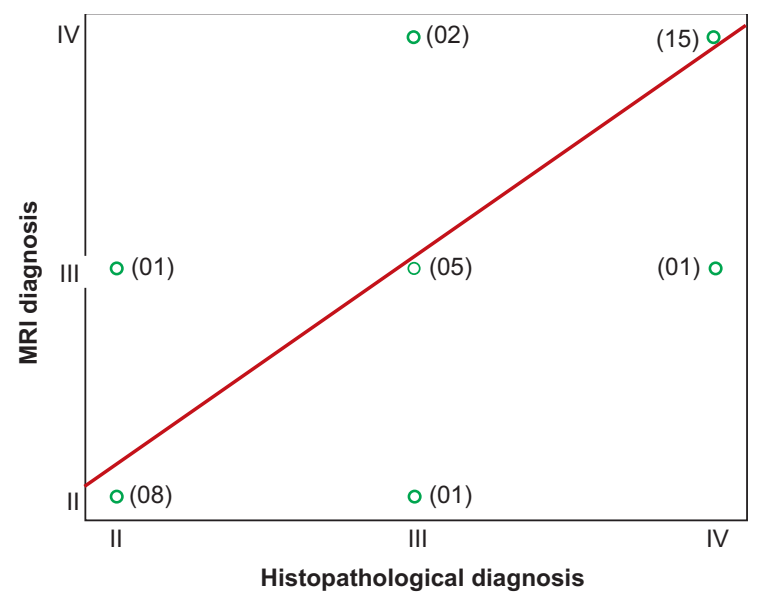

Fig.-2: Spearman's correlation coefficient was 0.889 and $p$ value was $<0.001$

Out of 33 patients, 09 patients were diagnosed as low grade gliomas by MRI, among them true positive were in 08 cases and 01 cases were diagnosed as low grade gliomas by MRI but was diagnosed as high grade gliomas by histopathology. This 01 case was false positive. Out of 33 cases of gliomas 24 were diagnosed as high grade gliomas by MRI, among them 23 cases were true negative and 01 case was diagnosed as high grade gliomas by MRI but was proved as low grade gliomas by histopathology. This 01 case was false negative. Shown in Table No. VI
4.7 Comparison between histopathological diagnosis and MRI diagnosis of patients of gliomas

Table VII

Comparison between histopathological diagnosis and MRI diagnosis patients of gliomas $(n=33)$

\begin{tabular}{lccc}
\hline MRI diagnosis & \multicolumn{2}{c}{$\begin{array}{c}\text { Histopathological diagnosis } \\
\text { (Gold standard test) } \\
\text { Low grade } \\
\text { (grade II) } \\
\mathrm{n} \mathrm{( \% )}\end{array}$} & $\begin{array}{c}\text { High grade } \\
\text { (grade III \& VI) } \\
\mathrm{n} \mathrm{( \% )}\end{array}$ \\
\hline $\begin{array}{l}\text { Low grade } \\
\text { (grade II) }\end{array}$ & $8(88.9)$ & $1(4.2)$ & $9(27.3)$ \\
$\begin{array}{l}\text { High grade } \\
\text { (grade III \& VI) }\end{array}$ & $1(11.1)$ & $23(95.8)$ & $24(72.7)$ \\
\hline Total & $9(100.0)$ & $24(100.0)$ & $33(100.0)$ \\
\hline
\end{tabular}

Table VII shows preoperative MRI diagnosis was correct with $93.9 \%$ accuracy. Sensitivity, Specificity, Positive predictive value and Negative predictive value of $\mathrm{MRI}$ in detecting tumor grade were $88.9 \%, 95.8 \%$, $88.9 \%, 95.8 \%$ respectively.

4.8 Validity test of MRI in detecting tumor grade of diffusely infiltrating supratentorial gliomas.

\section{Table-VIII}

Validity test of MRI in detecting tumor grade of diffusely infiltrating supratentorial gliomas.

\begin{tabular}{lccc}
\hline Validity test & $\begin{array}{c}\text { Estimated } \\
\text { Value (\%) }\end{array}$ & $\begin{array}{c}\text { 95\% confidence interval } \\
\text { Lower } \\
\text { Limit }\end{array}$ & $\begin{array}{c}\text { Upper } \\
\text { Limit }\end{array}$ \\
\hline Sensitivity & 88.9 & 58.6 & 99.0 \\
Specificity & 95.8 & 84.5 & 99.6 \\
Positive predictive value & 88.9 & 58.6 & 99.0 \\
Negative predictive value & 95.8 & 84.5 & 99.6 \\
Accuracy & 93.9 & 77.4 & 99.5 \\
\hline
\end{tabular}

Discussion:

Glial tumors constitute approximately $50 \%$ of newly diagnosed primary brain tumors, with lowgrade gliomas accounting for approximately 15\% of all brain tumors in adults ${ }^{10}$.

The grading of gliomas is needed for deciding clinical management and assessment of prognosis. The histologic classification of astrocytomas is complicated and inherent in all grading systems ${ }^{12}$ (Dean et al. 1990). Histological grading is a means of predicting the biological behaviour of a neoplasm. In the clinical setting, tumor grade is a key factor influencing the choice of therapies, particularly determining the use of adjuvant radiation and specific chemotherapy protocols ${ }^{11}$. 
The therapeutic management and prognosis in patients with gliomas depend on the reliable distinction between high and low-grade gliomas. Purpose of imaging in patients with brain tumors is the determination of the location, extent, type and malignancy of the tumor. Imaging is being used for primary diagnosis, planning of treatment including placement of stereotaxic biopsy, resection, radiation, guided application of experimental therapeutics and delineation of tumor from functionally important neuronal tissue ${ }^{12}$.

In AA and GBM, some characteristics overlapped. The different degrees of necrosis or cystic formation and increased abnormal vascularity seemed to be the key criteria in distinguishing GBM from $A A^{13}$.

CT and MRI are the mainstays of imaging in current practice. MR imaging with its multiplanar capabilities and superior contrast resolution is now modality of choice $^{7}$ (Knopp el al. 1999). MRI is very accurate in assessing the grade of gliomas. Tumor necrosis, irregular margins and peritumoral edema are most important indicators of gliomas grade ${ }^{5}$.

This study was conducted on 33 cases of supratentorial diffusely infiltrating astrocytomas at Department of Neurosurgery, Bangabandhu Sheikh Mujib Medical University, Dhaka, Bangladesh. The purpose of this study was to find out the correlation between MR imaging features with histological grading of supratentorial diffusely infiltrating astrocytomas.

In the present study, the age of the patients ranged from 18 years to 70 years. Mean age was 41.7 years. The highest incidence was in $<50$ years age group. Age incidence in intracranial gliomas is an important variable, which is observed varying from study to study. In present study, no particular age was found susceptible to develop gliomas.

Variation of gliomas with respect to sex is not also uncommon. The present study showed a male predominance $(75.75 \%)$ at the ratio of $3.12: 1$. About $74 \%$ of the 500 gliomas in the AlIMS study were male which is very close to this study ${ }^{14}$.

In this study regarding histopathological grading, $27.3 \%$ was grade II astrocytomas, $24.2 \%$ was grade III astrocytomas and $48.5 \%$ was grade IV astrocytomas.

In the present study, MRI features of gliomas in predicting grade included crossing mid line, peri- lesional edema, signal heterogeneity, hemorrhage, border definition, cystic formation or necrosis and mass effect of tumor.

Regarding crossing mid line $22.22 \%$ low grade astrocytomas, $25.0 \%$ anaplastic astrocytomas and $18.75 \%$ glioblastoma multiforme crossed the mid line. So, crossing mid line is not a good predictor of grading of gliomas. This result correlates with the study done by Asari et al. $(1994)^{13}$.

In this study regarding presence of peri-lesional edema, all of grade III and grade IV gliomas showed presence of peritumoral edema and maximum (81.25\%) of grade IV gliomas showed presence of marked peritumoral edema. So presence of peritumoral edema is important predictor of gliomas grading. Tervonen et al. (1992) ${ }^{15}$ demonstrated direct correlation between peritumoral edema with tumor grade $(p<0.01)$. Asari et al. $(1994)^{13}$ also found significant association between the perilesional edema and pathological grade of astrocytic gliomas. Present study also demonstrated significant association between peritumoral edema and $\mathrm{WHO}$ grade of intracranial gliomas.

The present study showed that all low grade gliomas had well circumscribed border in MRI. But $91.66 \%$ high grade gliomas had either poorly circumscribed border or highly infiltrating lesion. Asari et al. (1994) ${ }^{13}$ found significant association between definition of tumor border and the pathological grade of astrocytic gliomas $(p<0.02)$. This study also demonstrated significant association between tumor border definition \& WHO grading of gliomas.

This study showed maximum of marked mass effect $(91.7 \%)$ were histopathologically confirmed as grade IV gliomas and majority of mild to moderate mass effect $(58.6 \%)$ were confirmed as grade II gliomas. This study also showed significant association between the mass effect and the WHO grading of gliomas $(p=0.003)$. Dean at al. $(1990)^{9}$ found mass effect was the best predictor for assessing tumor grade $(p=.0000)$ using MR imaging. Asari et al. $(1994)^{13}$ also found significant association between the mass effect and pathological grade of astrocytic gliomas $(p<0.001)$.

Regarding necrosis/cystic change, this study showed $93.75 \%$ of necrosis/cystic change present cases were histopathologically confirmed as grade IV gliomas. Necrosis/cystic change were not present in MRI of 
grade II gliomas. So presence of necrosis/cystic change in MRI is good predictor of grade IV gliomas. Asari et al. (1994) ${ }^{13}$ found significant association between the necrosis or cystic change and pathological grade of astrocytic gliomas $(p<0.001)$. According to Dean et al. (1990) ${ }^{9}$ presence of cyst formation or necrosis in MR imaging was statistically significant $(p=0.05)$ for assessing tumor grade.

In present study, low grade gliomas characteristically were well defined; displayed no or little mass effect, peritumoral edema; nonenhancing or mild to moderate enhancing lesion on IV contrast administration; and were without necrosis/cystic changes. High grade gliomas were less well defined of poorly defined border; exhibited more mass effect, vasogenic edema; significant enhancing lesions; and majority presented with necrosis/cystic changes.

In this study preoperative MRI diagnosis was correct in 31 patients with accuracy of $93.9 \%$. Chishty et al. $(2010)^{5}$ showed preoperative MRI diagnosis was correct in 50 patients with accuracy of $94 \%$. Dean et al. $(1990)^{9}$ showed the overall accuracy rate of MR imaging was $83.3 \%$ for observer 1 and $80.6 \%$ for observer 2 for grading of astrocytic-series gliomas. Present study was very close to them.

In present study out of all 33 patients, 09 cases were diagnosed as low grade gliomas by MRI, among them 08 cases were true positive and 01 cases was diagnosed as low grade gliomas by MRI but was proved as high grade glioma by histopathology. This 01 case was false positive. Out of all 33 cases of gliomas 24 were diagnosed as high grade gliomas by $\mathrm{MRI}$, among them 23 cases were true negative and 01 case was diagnosed as high grade glioma by MRI but was proved as low grade gliomas by histopathology. This 01 case was false negative. Sensitivity, specificity, positive predictive value and negative predictive value of MRI in detecting tumor grade are $88.9 \%, 95.8 \%, 88.9 \%$ and $95.8 \%$.

Regarding correlation between Magnetic Resonance Imaging (MRI) findings with histopathological grading of patients with supratentorial diffusely infiltrating astrocytomas, Spearman's correlation coefficient is $0.889(p<0.001)$ which is statistically significant. So, Magnetic Resonance Imaging (MRI) findings of patients with supratentorial diffusely infiltrating astrocytomas correlate with histopathological grading.

\section{Conclusion:}

MRI is accurate in preoperative diagnosis and assessing the characteristics of intracranial gliomas. It is very accurate in assessing the grade of gliomas. Tumor necrosis, irregular margin, mass effect of tumor and peritumoral edema are most important indicators of tumor grade. The present study revealed that there is significant correlation between the MR imaging features and histopathological grading of intracranial gliomas.

\section{References:}

1. Gonzales M .'Classification and pathogenesis of brain tumors', in Brain tumors, $3{ }^{\text {rd }}$ edn, eds AH Kaye \& ER Laws, Jr, Elsevier, Sydney, 2011; 36-58.

2. Hassaneen W \& Sawaya .'Glioma resection', in Neurosurgery tricks of the trade cranial, eds R Nader, C Gragnaniello, SC Berta, AJ Sabbagh \& ML Levy, Thieme, New York, R 2014; 62-65.

3. López-Larrubia P, Cardenas EC, Metelo AM, Arias N, Maestro MM, Salguero A \& Cerdan S. 'Magnetic resonance imaging of gliomas', in Advances in the biology, imaging and therapies for glioblastoma, Intech, Rijeka, 2011; 225-250.

4. Kleihues P, Soylemezoglu F, Schauble B, Scheithauer BW \& Burger PC . 'Histopathology, Classification, and Grading of Gliomas', Glia, 1995;15:211-221.

5. Chishty IA, Rafique MZ, Hussain M, Akhtar W, Ahmed MN, Sajjad Z \& Ali SZ. 'MRI characterization and histopathological correlation of primary intra-axial brain glioma', JLUMHS, 2010 ; 09:, 02: 64-69.

6. Panigrahy A, Marvin D, Nelson J \& Bluml S. 'Magnetic resonance spectroscopy in paeditric neuroradiology: clinical and research application', Pediatr Radiol, 2010; 40: 3-30.

7. Knopp EA, Cha S, Johnson G, Mazumdar A, Golfinos JG, Zagzag D, Miller DC, Kelly PJ \& Kricheff II. 'Glial neoplasm: Dynamic contrast-enhanced T2-weighted MR imaging', Radiology, 1999; 211: 791-798.

8. Daumas-Duport C, Scheithauer B, O'fallon J \& Kelly P. 'Grading of astrocytoma', Cancer, 1988; 62:2152-2165.

9. Dean BL, Drayer BP, Bird CR, Flom RA, Hodak JA, Coons SW \& Carey RG. 'Gliomas: Classification with MR imaging', Radiology, 1990;174: 411-415.

10. Sanai N \& Berger MS. 'Low grade and High grade Gliomas', in Principles of Neurological Surgery, eds RG Ellenbogen, SI Abdulrauf \& LN Sekhar, 2012, Elsevier Saunders, Philadelphia, 2012;527-534.

11. Louis DN, Ohgaki H, Wiestler OD, Cavenee WK, Burger PC, Jouvet A, Scheithauer BW \& Kleihues P. 'The 2007 WHO Classification of Tumours of the Central Nervous System', Acta Neuropathol, 2007;114: 97-109.

12. Jacobs AH, Kracht LW, Gossmann A, Ruger MA, Thomas AV, Thiel A \& Herholz K. 'Imaging in Neurooncology', NeuroR, 2005; 2: 333-347.

13. Asari $\mathrm{S}$, Makabe $\mathrm{T}$, Katayama $\mathrm{S}$, Itoh $\mathrm{T}$, Tsuchida $\mathrm{S}$ \& Ohmoto T. 'Assessment of the pathological grade of astrocytic gliomas using an MRI score', Neuroradiology, 1994; 36: 308-310.

14. Easwaran R. 'Supratentorial Astrocytomas', in Textbook of Neurosurgery, eds PN Tandon \& R Ramamurthi, Jaypee Brothers, New Delhi, 2012; 3: 1495-1527.

15. Tervonen O, Forbes G, Scheithaurer BW \& Dietz MJ. 'Diffuse fibrillary astrocytomas: correlation of MRI features with histopathologic parameters and tumor grade', Neuroradiology, 1992; 34:173-178. 